\title{
Central Pancreatectomy for Pseudo Papillary Neoplasm Of Body of Pancreas
}

\author{
Dr.R.Jayakumar ${ }^{1}$,Dr.D.N Renganthan ${ }^{2}$ Dr.R.Radhika ${ }^{3,}$ \\ Dr.D.J.Balaji ${ }^{4}$ Dr.P.Viggnesh ${ }^{5}$, \\ Dr. J. Sathish Kumar ${ }^{6}$ Dr. P. T. Mohamed Fayiz ${ }^{7}$ Dr. S. Pandiyaraj ${ }^{8}$ \\ Dr.Muhammed Owaise ${ }^{9}$ Dr.S. Vignesh Shankar ${ }^{10,}$ Dr.A.Balamurugan ${ }^{11}$
}

\begin{abstract}
Pancreatic neoplasms might be benign or malignant. Adenocarcinoma is the most common type of malignancy. Solid pseudopapillary neoplasm constitutes about $<1-2 \%$ of pancreatic neoplasm. It usually arises from head and tail of pancreas. They are usually asymptomatic. Diagnosed by CT scan of abdomen. The pathognomonic feature in histological diagnosis is presence of pseudo papillary arrangement of bland appearing tumor cells. They are usually benign with $20 \%$ low grade malignant potential. They have very good prognosis compared to Adenocarcinoma even in the presence of distant metastasis with surgical resection. Here , We present a case of 20 year old female with dull aching epigastric pain with CT scan showing a well encapsulated mass arising from body of pancreas. After the necessary investigations patient underwent, Central pancreatectomy, A rarely done procedure for encapsulated tumors of body of pancreas which prevents post operative exocrine and endocrine insufficiency and also the complications of extensive pancreaticoduodenectomy / splenectomy.
\end{abstract}

\section{Introduction}

Solid Pseudopapillary neoplasm of pancreas is a rare tumor comprising $<1-2 \%$ of exocrine pancreatic tumors. It is almost exclusively seen in females at $2^{\text {nd }}$ and $3^{\text {rd }}$ decades of life. It is usually present with asymptomatic mass / accidentally diagnosed during imaging studies.

\section{Case report :}

A 20 year old female came with complaints of epigastric pain not relieved by taking PPIs for 6 months. No history of vomiting / hematemesis. On examination, Patient had a palpable mass in the epigastric region of about $9 \times 8 \mathrm{~cm}$. Firm in consistency and not mobile.

\section{Investigations}

CT scan - A well encapsulated mass of about $9.4 \times 7.2 \mathrm{~cm}$ arising from the body of pancreas.

OGD scopy - Normal

CA 19-9 / Amylase / Lipase - Within normal limits.
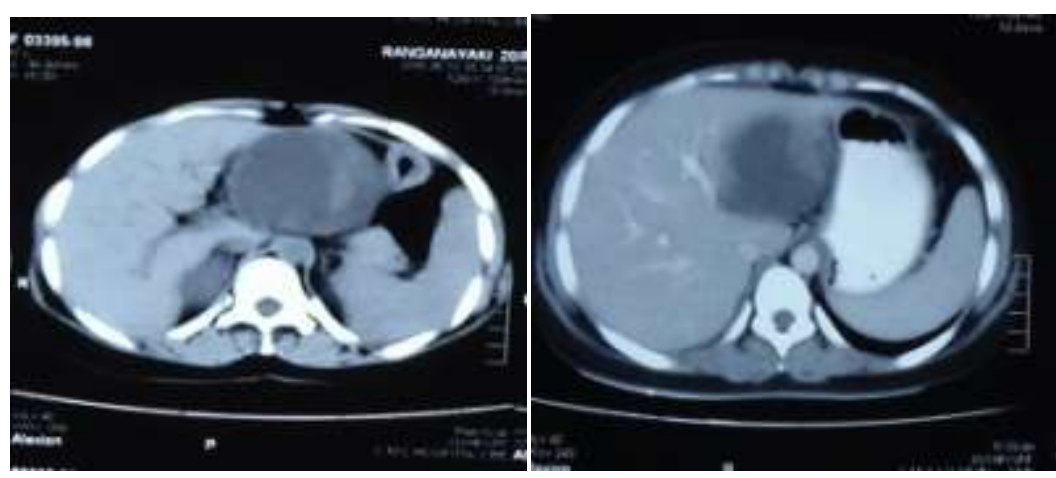

III. Management

Patient underwent Laparotomy and Central pancreatectomy done.

Surgical technique : 
Under General anesthesia , patient in supine position , parts painted and draped. Bilateral subcosatal incision made and laparotomy done. Lesser sac approached. A 9 x $8 \mathrm{~cm}$ well defined encapsulated mass seen arising from body of pancreas. Pancreas mobilised from the bed. A linear cutter stapler applied at the junction of body and head of pancreas. Tumor along with the body of pancreas mobilized. Distally $1 \mathrm{~cm}$ from the tumor pancreas divided. Specimen retrieved out and sent for HPE. Duct cannulated. End to side roux en Ypancreaticojejunostomy done by Dunking technique. After achieving complete hemostasis. Drain kept. Abdominal wound close in layers.

Post operative period uneventful.drain removed on $6^{\text {th }}$ POD and patient discharged on $8^{\text {th }}$ POD.

GROSS APPEARANCE:
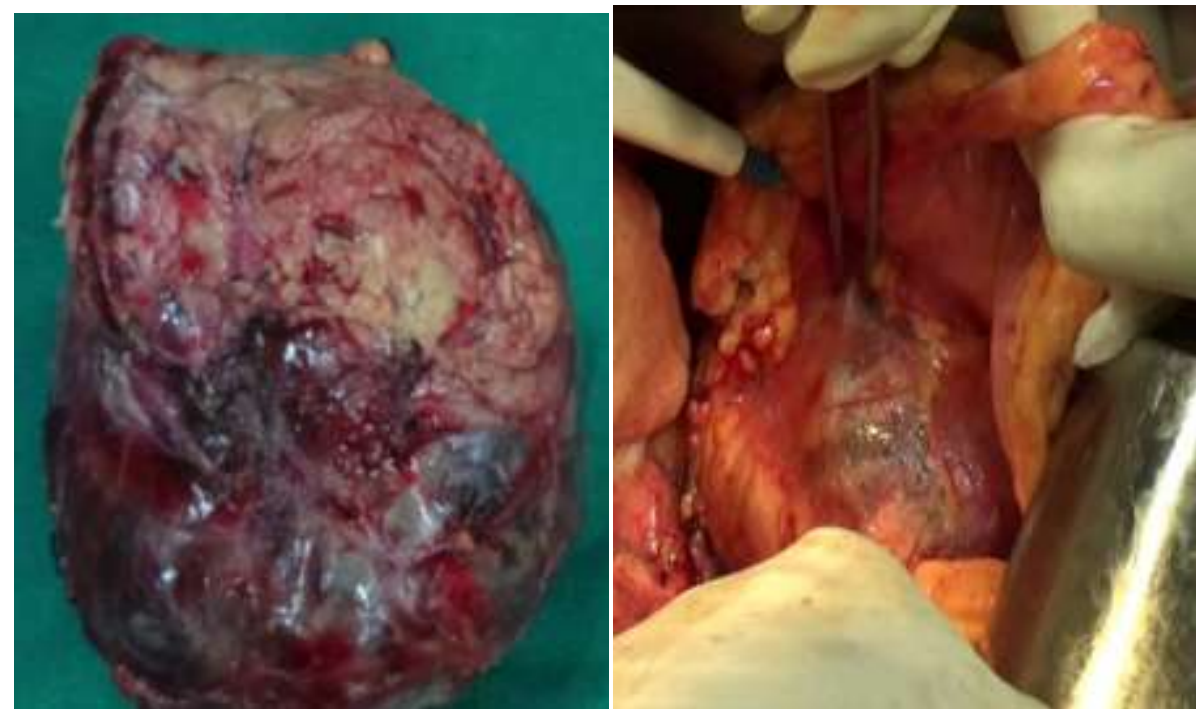

Histopathology :

HPE showed as Solitary pseudopapillary neoplasm of pancreas with Vimentin and S-100 and Cytokeratin positivity. The cut margins of specimen are negative.

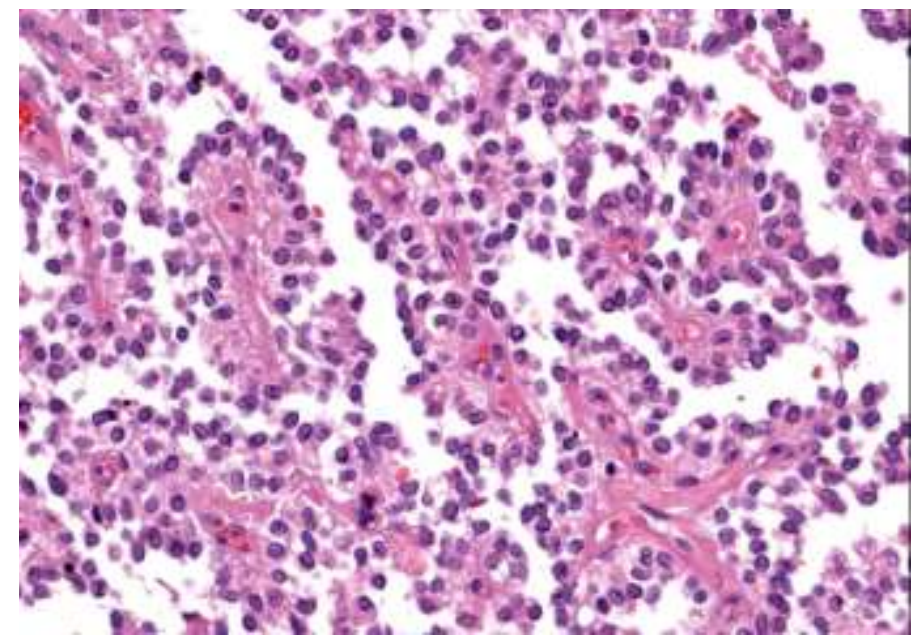

\section{Discussion}

Solid pseudopapillary neoplasm of Pancreas is a rare entity comprising of $<1-2 \%$ of exocrine pancreatic tumors. It is almost exclusively seen in females at $2^{\text {nd }}$ and $3^{\text {rd }}$ decade of life. It is often benign tumor with $20 \%$ showing low grade malignant potential. The tumor usually presents as an asymptomatic mass or diagnosed accidentally during imaging studies. Some patients present with dull aching abdominal pain in the epigastric region.

\section{Investigations}

CT scan is the investigation of choice and shows a well defined encapsulated lesion with heterogenous enhancement. FNAC can be done and shows epitheloid cells with fibrovascular cores. No evidence of 
pleomorphism or mitotic activity. Pathognomonic finding is Pseudopapillary arrangement with bland appearing tumor cells. Immunohistochemistry shows reactivity for Vimentin, Anti trypsin, Cytokeratin , S-100 protein and Neuron specific enolase.

\section{Treatment:}

Surgical resection is the treatment for Solid Pseudopapillary neoplasm. If tumor is too large in size or adherent to adjacent structures, Neo adjuvant chemotherapy / Radiotherapy can be given. With surgical excision it has good prognosis even with distant metastasis.If Post operative HPE shows that the tumor is malignant one, Adjuvant Chemotherapy is given.

\section{Central pancreatectomy:}

It is also referred to as median or segmental pancreatectomy. It is reserved for benign and low grade malignant tumours of middle pancreas.It can be done only whenremenent pancreas tissue is atleast $5 \mathrm{~cm}$ distally.The advantages of central pancreatectomy over pancreaticoduodenectomy / distal pancreatectomyare there is no exocrine or endocrine insufficiency,normalgastroduodenalbiliary continuity and normal pancreatic parenchyma is preserved and absence of post splenectomy complications. The main complication of central pancreatectomy is pancreatic fistula.

\section{Conclusion}

Large encapsulated tumors in pancreas are usually benign in nature and can be treated with limited surgical resection. In this case, As the tumor is confined to body of pancreas central pancreatectomy was done which prevents exocrine and endocrine insufficiency and postsplenectomy complications post operatively. But for adenocarcinoma or other high grade malignancies it is advisable to carryout radical surgical procedures.

\section{Referance}

[1]. Dagadi A, SerioG.Pancreatectomiaintermedia. Vol XI , 1984, p 850

[2]. Rotman N Sastre B, Fagnioez PL. Medial pancreatectomy for tumors of neck of pancreas1993;113:532

[3]. Maria A. Johnson ,ShanmugasundaramRajendran . Central pancreatectomy for benign pancreatic pathology; anzJ . Surg.2006 ; 76:987-995

[4]. Blumgart surgery of the liver biliary tract and pancreas; 5E ; vol 1: 945-959

[5]. Claudio bassi . Middle segment pancreatectomy : A useful tool in management of pancreatic neoplasms ; J gastrointestsurg (2007) 11: $421-424$ 